\title{
El autor imperceptible en la poesía de Jorge Teillier*
}

Theimperceptible author in the poetry of Jorge Teillier

\section{Pedro Aldunate $F^{* *}$}

Universidad de Concepción, Chile

E-mail: pedroaldunate@hotmail.com

\section{RESUMEN}

Este artículo presenta una lectura espacial en la que se marca un desplazamiento o devenir a través de los textos poéticos. La lectura se propone como viaje a través de la textualidad poética. Espećificamente, se trazan los desplazamientos de los sujetos de la enunciación, los que señalan, por su parte, una determinada figura de autor que se revela, como huella, a través de los signos aparentes de su desaparición. A partir del diálogo con los textos de Jorge Teillier, se introduce la noción crítica del autor imperceptible.

Palabrasclaves Autor, construcción autorial, figura del autor, poeta, devenir, espacio, línea de fuga, desterritorialización, devenir imperceptible, afuera, huella, literatura menor, poe sía lárica, lar.

\section{ABST RACT}

This article presents a spatial reading where a displacement or becoming through poetic texts is stamped. The reading is proposed as a journey through poetic textuality. Specifically, we trace the displacements of the subjects of enunciation, displacements which thus signal a specific authorial figure who reveals himself as a tracethrough the apparent signs of his disappearance. Starting from the dialogue with Jorge Teillier's texts, the critical notion of the imperceptible author is introduced.

Keywords Author, authorial construction, author's figure, poet, becoming, space, fugueline, deterritorialized, imperceptible becoming, outside, trace, minor literature, larical poetry, lar. Recibido: 27-04-2006. Aceptado: 15-10-2006.

* Este artículo fue realizado para el seminario "Poesía chilena contemporánea", dirigido por el D r. J uan Zapata G., en el marco del programa de D octorado en Literatura Latinoamericana, Universidad de Concepción, año 2005. 


\section{INTRODUCCION}

V ISU ALIZO en la siguiente reflexión de la poesía de Jorge Teillier ${ }^{1}$ un desplazamiento que conecta ciertasfiguras poéticastextuales y ficcionales-suje tos de la enunciación y personajes- con el autor entendido como proceso de construcción autorial ${ }^{2}$. Se concibe la lectura de la obra de Teillier de manera espacial y se propone participar - como lector- de un viaje dentro de la ficción literaria ${ }^{3}$ hasta sus propios límites en tanto arte de la palabra o poesía. Se sitúa así un devenir a través de la textualidad poética, un recorrido que se aproxima al silencio como límite de la palabra poética. El objetivo de este trabajo es, entonces, recorrer un periplo a través de la escritura de ciertos textos; escritura que, por su parte, reescribe y actualiza la figura del poeta, cuestionando tanto el sentido de la práctica poética como la propia condición y construcción del autor a través de su obra4

${ }^{1}$ Teillier, Jorge (1953-1996), poeta chileno perteneciente a la generación del '50. Sobre esta generación se han referido variosautores (M uñoz y 0 elker, 1995). Lasfiguras más representativas de esta generación son Enrique Lihn, Jorge Teillier, Armando U ribe, Efraín Barquero, Alberto Rubio, C arlos de Rokha, Fernando G onzález Urízar, Jorge Cáceres, Stella D íaz Varín, entre otros.

2 Empleo el concepto de autor en tanto proceso de construcción autorial, a partir de la idea desarrollada por el Dr. M iguel Gomes S., en el seminario "Los mitos del autor en la literatura latinoamericana", U niversidad deC oncepción, Escuela de Verano 2003. Sobre el concepto y problema del autor, ver Roland Barthes (1981), especialmente en lo que respecta a la idea de "muerte de autor". Sobre la "desaparición del autor" y sobre losespacios queesta desaparición hace aparecer, ver M ichel Foucault (1999), ensayo fundamental que abre el concepto - desde una perspectiva críticay que permite distintas preguntas y figuraciones posibles del autor entendido en tanto función de discurso. Sobre la relación entreel autor y la obra, establezco diálogos en torno a lo que puedo llamar una "ética del autor" planteada por M ichel Foucault (1999) y por M aurice Blanchot (1993), según la cual el autor se construye como tal a partir de su obra y en un proceso recíproco. Una primera aproximación al problema del autor se encuentra en mi tesis de $M$ agíster en Literatura H ispanoamericana "La construcción autorial deArmando U ribe Arce: el autor, la obra y la muerte", Universidad de Concepción, 2004. Por último, para situar el concepto y su función, ver Santibáñez (2004) síntesis "N otas sobre el problema autor y su función".

${ }^{3}$ Con el concepto de ficción (del latín fingo, configuro, doy forma) me refiero a aquella "forma del discurso que hace referencia a personajes y cosas que sólo existen en la imaginación de su autor, y luego en la del lector/espectador" (Pavis, 1998: 206). En este sentido el autor, como el actor teatral, es también un simulador, ya que muestra y oculta un determinado "estatuto de verdad" a través de un "acto ilocutorio" que rige el discurso.

"En este sentido la obra pone en escena figuras de autor. U tilizo la noción de "figura de autor" en dos sentidos: el autor en tanto figura teatral, es decir, como cuerpo-autor; y en tanto figura discursiva, es decir, como figuración de un cuerpo-autor "presente" de alguna forma en el texto, incluso en el signo de su desaparición. Así, el proceso de construcción autorial dibuja una figura, la que está en constante movimiento y creación desí misma. La figura de autor es de este modo teatral, ya que lleva a cabo desplazamientos en los que se descubren posibles sentidos, tal como sucede con la figura dramática. Al respecto, traslado la noción de figura dramática utilizada por M arta Contreras al espacio de la literatura, para visualizar los movimientos que los autores realizan en una escena 
Para efectos de análisis crítico trabajo con la perspectiva o mirada escénica de los textos poéticos, situando en ellos un determinado desplazamiento que conecta las categorías del autor y de la obra. D e este modo se proponela posibilidad de transgredir la dicotomía epistemológica autor-obra y también, en cierto sentido, aquella que separa la ficción de la realidad designada. Sigo en este punto a Jacques D errida (1971), cuyas ideas permiten deconstruir "Ia vieja reji"la" quesuponela existencia decategorías absolutas que dividen, por ejemplo, el autor de la obra literaria. Para establecer esta lectura propongo diálogos con ciertos autores asociados al posestructuralismo, entre ellos, M ichel Foucault, Gilles D el euze, Félix G uattari y Jacques D errida. Específicamente, para situar la poesía de Teillier en el contexto de la escena literaria chilena me refiero al concepto de literatura menor (D eleuze y Guattari, 1990; D eleuze, 1994; D eleuze y Guattari, 1997).

Como objeto de reflexión de este ensayo se ha escogido una serie de poemas - o fragmentos de la obra- que conforman distintos espacios en la obra de J orge Teillier. Como se verá, la elección de éstos no responde a un orden cronológico de aparición o publicación, los textos escogidos aparecerán según el desarrollo mismo del objeto de reflexión. Conformeal carácter ensayístico de esta lectura, se establecerá como objetivo específico la necesidad de producir un texto que dialogue con los textos poéticos, es decir, otro texto que dé cuenta de ciertos momentos de esta poesía y que a su vez recorra el camino que trazan estos poemas, ello con el fin de identificar y describir los desplazamientos de los sujetos de la enunciación en el espacio de esta poética; y la configuración de una determinada figura imperceptible de autor, rastreable en el texto aun en el signo aparente de su desaparición ${ }^{5}$.

literaria 0 en el espacio ficcional de la literatura. La figura del autor, en este sentido, corresponde a una "asociación dinámica de elementos; a un modelo, diseño, forma o esquema inferible (...); a un personaje en particular cuya apariencia y desplazamiento constituye a su vez una figura visual". La noción defigura, desde esta perspectiva, también nos permite explorar cómo la misma construcción autorial constituye una construcción ficcional que permite ciertas representaciones del autor, en donde éste, por ejemplo, aparece interpretando a un personaje o realiza acciones en tanto él mismo (performer). Por otra parte, losmedios masi vos de comunicación enfatizan el carácter visual y teatral dela figura de autor, al difundir la imagen del cuerpo-autor. Sobrela noción de figura ver Contreras (1994: 4, 8, 33).

${ }^{5}$ D iscuto desdeFoucault (1999) la idea de "muerte de autor" planteada por R oland Barthesen sus distintos análisis sobre el discurso literario (Barthes, 1981). Foucault no habla de "muerte del autor" sino de "desaparición". Según Foucault esta desaparición del autor proyecta un espacio quese puedelocal izar. En este sentido propongo - con D eleuze y G uattari- la noción de autor imperceptible, precisamente, en tanto la desaparición del autor va dibujando una determinada figura del autor que describe y con un determinado proceso de construcción de autor. 


\section{LA POESIA LARICA COMO UNA LITERAT URA MENOR}

JorgeTeillier señala en su ensayo "Los poetas de los lares" (1999) la aparición de un grupo relativamente homogéneo de poetas vinculados a la generación del '50. D entro de esta generación surge un movimiento que posee una línea característica que se denomina poesía de los lares. U na primera característica de estos poetas es el hecho de que "vuelven a integrarse al paisaje, a hacer la descripción del ambiente que los rodea" (... ) "los poetas nuevos han regresado a la tierra, sacan su fuerza de ella" (Teillier, 1999: 22). Se habla, entonces, de un regreso al lugar del origen, de la niñez, por la nostalgia de un tiempo perdido, a la vez que se advierte un regreso de la ciudad al campo, "un rechazo a veces inconsciente a las ciudades, estas megápolis que desalojan el mundo natural" (Teillier, 1999: 22). En este sentido, la poeśa de los lares propone, aunque no mediante un plan previo explícito, una resistencia al proyecto de modernización del hombre en nuestro tiempo.

La modernidad, señala García C anclini, se compone de cuatro proyectos, a saber: proyecto emancipador, expansivo, renovador y democratizador (G arcía Canclini, 1998). Es a este proyecto moderno al cual se opone esta llamada poe sía de los lares, sobretodo en sus ideales expansivo y renovador, no declarándose como un movimiento de ruptura, explícitamente crítico de la realidad, ni con una intención vanguardista, sino creando una línea de fuga, en este caso, al estado sitiado del hombre en nuestras sociedades y al estado sitiado de la poesía chilena. De modo que la poesía lárica se establece como crítica de la modernidad y en este sentido puedeser considerada en tanto poesía posmoderna, ya que se establece una resistencia a la condición espacio-temporal originada en la primera modernidad.

De esta forma, la poeśa de los lares, o más específicamente el término lar, nace como oposición al orden establecido de las megápolis modernas y posmodernas, cuya concepción del tiempo y del espacio, así como de la vida, condicionan la existencia del sujeto. Jorge Teillier explicita de esta manera la poeśa de los lares:

Frente al caos de la existencia social y ciudadana los poetas de los lares (sin ponerse de acuerdo entre ellos) pretenden afirmarse en un mundo bien hecho, sobre todo en el mundo del orden inmemorial de las aldeas y los campos, en donde siempre se produce la misma segura rotación de las siembras y cosechas,

${ }^{6}$ Jorge Teillier incluye una gran cantidad de autores que se adscribirían a esta poética. Entre los másimportantescabemencionar, en primer lugar, al mismo Teillier, a Efraín Barquero, Pablo Guíñez, Alberto Rubio, Rolando Cárdenas, Alfonso Calderón, entre otros. 
de sepultación y resurrección, tan similares a la gestación de los dioses (recordemos a D ionisos) y de los poemas (Teillier, 1999: 25).

Los poetas de los lares configuran un nuevo espacio, un nuevo territorio. Así entendido, el lar constituye un encuentro del hombre consigo mismo, es decir, con sus raíces ancestrales, un retorno al "no tiempo" mítico, al eterno ciclo de la natural eza y el hombre, de la vida y la muerte. El mismo concepto lar designa en sus orígenes el lugar de la casa donde está el fuego, donde se reúne la familia, donde se invoca a los dioses. N o se trata, como señala N iall Binns, quien cita a su vez a Teillier, de una "vuelta al descripci onismo de la poesía criollista, sino una especie de 'realismo secreto', una visión del mundo como 'un depósito de significados y símbolos ocultos" (Binns, 2001: 32). Este espacio constituye un centro, el lugar primordial del hombre, que en nuestras sociedades actuales es desintegrado. Por ello el lar se entiende como una aldea en las márgenes, un territorio limítrofe, fuera del orden dela urbey de la vida dela ciudad posmoderna ${ }^{7}$. Se verá más adelante cómo esta al dea es más que limítrofe y cómo el sujeto o los sujetos que la habitan se encuentran siempre en tránsito hacia otro espacio, en un perpetuo devenir. D e esta forma, el lar o la al dea lárica constituye la configuración de un espacio que desterritorializa al hombre cercado en la ciudad, en la urbe contaminante y bulliciosa. Los poetas de los lares trazan una línea de fuga, una resistencia al orden alienante de la ciudad moderna o posmoderna, y en este sentido, cumplen las características señaladas por D eleuze y G uattari, respecto de una literatura menor.

Ahora bien, esta creación de un espacio nuevo no sólo es una configuración de un territorio que se resiste al espacio cercado y al orden alienante de la ciudad. La poesía deloslaresse establecetambién como una literatura menor, puesto que desterritorializa el proyecto literario moderno de las primeras décadas del siglo XX, cifrado en la altisonante vanguardia de los años 30. D e esta forma la nueva poesía de la generación del ' 50 se establece como una literatura menor respecto de un proyecto de literatura mayor ya concretado en nuestra tradición poética. D icha literatura mayor estaría representada por las figuras paradigmáticas de N eruda, H uidobro y $M$ istral, principalmente, y de D e Rokha y Parra, en un cierto sentido. Al respecto, señala Teillier quelos poetas no son ya "el centro del universo", "sino que son observadores, cronistas, transeúntes, simples hermanos de los seres y las cosas" (Teillier, 1999: 24).

${ }^{7}$ En cierto sentido la proposición lárica puede ser léda como un ejemplo dearcaísmo posmoderno, ya que implica una regresión a ciertas costumbresy saberes arcaicos. La idea dearcaí smo posmoderno ha sido planteada por el D r. Juan Zapata G. como característica de la condición posmoderna. 
En cuanto a las características señaladas por D eleuze y G uattari (D eleuze y Guattari, 1990 ) respecto de una literatura menor se puede señal ar lo si guiente 8 . Señalan los autores que "una literatura menor no es la literatura de un idioma menor, sino la literatura queuna minoría hace dentro de una lengua mayor" (D eleuze y G uattari, 1990: 28). Así, los llamados poetas de los lares constituyen la expresión de una minoría, aquella de los poetas de provincia, los poetas de las aldeas rurales, los poetas de La Frontera. Ahora bien, no sepercibeen Teillier un grado extremo de desterritorialización del idioma, aunque sí una recuperación de un registro propio del lenguaje de la vida rural. Al respecto, se puede decir que la poesía de los lares toma elementos de la antipoesía parriana, puesto que redescubre el lenguaje simple y directo de la vida del campo, pero dejando a un lado el carácter desacralizador de la antipoesía de Parra. En cuanto a la segunda característica señalada por los autores, se podría afirmar, en primer lugar, que en la poesía deTeillier nada hay de un discurso político. Sin embargo, una segunda lectura revela la conformación de una crítica (a veces implícita y a veces explícita) que es, a la vez, política, social, económica y cultural. M ás aún, la conformación del lar como un nuevo territorio que desterritorializa el orden político, social, económico y cultural imperante supone un nuevo orden, una nueva casa real e imaginaria, es decir, una nueva micropolítica. $D$ e esta forma los sujetos y los personajes que figuran la voz del poeta - de la construcción autorial Jorge Teillier- trazan una línea de fuga, una resistencia. A ellos les está dada la posibilidad siempre difícil y, también, como se verá, siempre precaria, del retorno a la aldea lárica.

La tercera característica es que en literatura menor "todo adquiere un valor colectivo". La proposición del lar como espacio desterritorializante supone "Ia posibilidad de expresar otra comunidad potencial, de forjar los medios de otra conciencia y de otra sensibilidad" (D eleuze y G uattari, 1990: 30). En este sentido, el lar sería el espacio donde se hace posible forjar esa otra conciencia y esa otra sensibilidad. El lar sería la al dea de los poetas o el lugar delos metecos, como diría Linn.

El lar se constituye entonces como un espacio en donde el hombre alienado de la ciudad encuentra la posible salud, el retorno a la vida y a la voluntad de la naturaleza. "La salud como literatura, como escritura, consiste en inventar el pueblo que falta" (D eleuze, 1994: 15), ese pueblo no es otra ciudad, estructurada y jerarquizada, en este caso, por el cuestionado proyecto moderno, es un pueblo "en perpetuo devenir, siempre inacabado".

${ }^{8}$ Tres son las características de una li teratura menor según $D$ el euze y Guattari, a saber: la desterritorialización delalengua, la articulación de lo individual en lo inmediato político y el dispositivo colectivo de enunciación. 


\section{LOS DESPLAZAMIENTOS DE LOS SUJETOS EN LA POESIA DE JORGE TEILLIER}

Seguir la línea de fuga que trazan los sujetos láricos supone abandonar la noción tradicional del lector, supone abandonar, a su vez, cierta condición espacial y territorial. El lector es entonces, en esta poesía, el sujeto que recorre las líneas de fuga trazadas por los sujetos del texto. El lector es el sujeto que se abandona totalmente en el viaje de la literatura, para devenir finalmente imperceptible, en la medida que la propia ficción, con sus múltiples sujetos creados, deviene imperceptible. Para seguir losmovimientos desterritorializantesvisualizaremosla poesía deTeillier en tanto espacio que constantemente es desterritorializado. Así entendido, el lector forma parte del dispositivo colectivo de enunciación, puesto que al seguir la línea de fuga trazada por la escritura participa en la recreación de ésta y en la resistencia que se propone.

Puesto que no se hará referencia a un solo texto poético, es necesario tomar en cuenta la noción de intertextualidad, que a su vez constituye un espacio, entre los textos. La noción de intertextualidad se entiende como "la interacción de textos que se produce en el interior de un solo texto. Según esto, el lenguaje poético aparece como un diálogo de textos: toda secuencia está doblemente orientada, hacia un acto de reminiscencia (evocación de otro texto) y hacia un acto de sumación" (K risteva, 1981: 167) ${ }^{9}$. Los textos escogidos dialogan entre sí, representan distintos momentos y estados de una poética, configuran un espacio, es decir, conforman una red intertextual. Para seguir estos movimientos desterritorializantes se propone observar el conjunto de textos como puntos de un mapa, como lugares dentro de un macroespacio, donde a la vez se construyen y deconstruyen ciertos proyectos espaciales trazados por una determinada figura y construcción de autor.

El primer desplazamiento es la proyección y el tránsito hacia el lar. El segundo de ellos es aquel que desterritorializa el proyecto mismo del lar y que propone el llamado devenir imperceptible, en donde es posible visualizar una figura distinta del autor, no ya el Ilamado poeta lárico.

En el poema "Juegos" tenemos a un sujeto que expresa la posibilidad de un deseo. En la ciudad el sujeto recuerda los juegos de la infancia, se da cuenta de que el niño es quien quierey puedejugar. El hombre, atrapado en su conciencia temporal, permanece solo y triste: "los grandes están de piejunto a la luz ruinosa de la tarde" (Teillier, 1992: 44); el niño es quien le recuerda al hombre la muerte; el niño juega con la muerte, mientras el hombre no puede esconderse de ésta, puesto que ha perdido toda conciencia lúdica, toda conciencia atemporal. $\mathrm{N}$ o hay aquí movimiento, hay una certeza dela irreversibilidad del tiempo y del espacio, una certeza del tiempo perdido dela infancia. La conciencia del tiempo 
lineal, del tiempo que no se muerde la cola, como también la condición del hombre en la ciudad moderna, terminan aprisionando a este sujeto-personaje: "para los grandes sólo llega el silencio / vacío como un muro que ya no recorren sombras" (Teillier, 1992: 44). El silencio no constituye aquí un devenir imperceptible, el silencio es analogía de la muerte. El retorno a la niñez es una posibilidad irrealizada, un deseo expresado queno al canza el devenir, queno al canza el no ser entrelo que somos y lo que deseamos. Se configura, en este primer espacio escénico, un sujeto que nos habla y que representa la condición desolada y deshumanizante del hombre posmoderno a partir de la segunda mitad del siglo $X X$. D e esta condición el sujeto huye, va hacia los espacios limítrofes de la ciudad, rechaza la vida de la urbe, la vida estructurada y al ienante de la ciudad. En ese tránsito explota, necesariamente, la conciencialineal del tiempo, así como la asociación con la ciudad como centro territorial. El sujeto transita ahora hacia los lindes, se sienta en las estaciones de la ciudad a esperar un tren, es ahí donde comienza a trazar el primer movimiento desterritorializante.

El sujeto está en una estación de trenes, esperando un tren hacia otro lugar. El sujeto del poema "Andenes" dice: "te gusta quedarte en la estación desierta / cuando no puedes abolir la memoria" (Teillier, 1992: 44); este sujeto está aún en la ciudad, pero en sus límites, en una estación de trenes. El tiempo continúa con su "tictaqueo", siempre hacia un futuro incierto que es necesario construir: "el reloj sigue diciendo / que la noche es el único tren / que puede llegar a este pueblo, / y a ti te gusta estar inmóvil escuchándolo" (Teillier, 1992: 44). Se visualiza a un sujeto que espera, que aún no se despide de la ciudad en que habita; se visualiza a un sujeto que no tiene un destino, que permanece inmovilizado escuchando el "tictaqueo" del reloj, esperando un tren que aún no llega. En "Andenes" hay sólo posibilidad de una fuga, de un tren hacia el afuera.

La posibilidad de la fuga se hace real a partir del poema "D espedida". En este texto el sujeto se despide de la ciudad, del mundo de la urbe, se despide para iniciar su viaje, su retorno al lar que se proyecta en el afuera: "M e despido demi mano / que pudo mostrar el paso del rayo, ( ... ) , me despido del papel blanco y de la tinta azul, ( ... ) , me despido de la memoria / y me despido de la nostalgia / - la sal y el agua- / de mis días sin objeto" (Teillier, 1992: 38-39). D e este modo, el sujeto abandona la ciudad y la conciencia del tiempo, pero, más aún, el sujeto - en este momento un signo huella del autor- se despide de su propia palabra poética: "y me despido de estos poemas: / palabras, palabras - un poco deaire/ movido por los labios- palabras/ para ocultar lo único verdadero: / que respiramos y dejamos de respirar" (Teillier, 1992: 38-39). El sujeto aún está en la estación que, como se ha dicho, es un punto de partida. En esta estación, el sujeto acaso intuye por primera vez lo imperceptible, el silencio que está en el 
afuera, más allá "del papel blanco y de la tinta azul". A partir de "D espedida" se abre la posibilidad al primer movimiento desterritorializante.

Se visualiza ahora a un sujeto en movimiento, un sujeto que transita hacia otro lugar, que busca situar un territorio a partir de una conciencia diferente. Ese espacio se proyecta como el lar, el territorio que desterritorializa la concepción territorial dela ciudad posmoderna. En este tránsito hacia el lar el sujeto es un forastero. Antes dellegar el sujeto sepropone construir una casa, en el futuro pueblo que lo acogerá: “los gallos me despiertan / y sus cantos / prometen ayudarme a alzar la casa" (Teillier, 1992: 62 ). Esa casa es la proyección del lar.

El primer movimiento desterritorializante se constituye, entonces, en la creación de la al dea lárica. En ese lugar la posibilidad dela felicidad sehace real, "un día u otro / todos seremos felices" (Teillier, 1992: 90); Ia conciencia lineal del tiempo es remplazada por una conciencia atemporal: "N o sabremos / si la caja demúsica / suena durante horas o un minuto" (Teillier, 1992: 90). El sujeto del poema "Edad de oro" propone la posibilidad de una nueva condición existencial, quesupera la concepción occidental del tiempo eincluso hasta de la muerte: "nos saludaremos sonriendo apenas / pues todavía creeremos estar vivos" (Teillier, 1992: 90). Esla apuesta por el "pueblo feliz", el pueblo que siempre se está construyendo en las márgenes del territorio en que se habita y se permane $\mathrm{ce}^{10}$. En este espacio, se observa al sujeto reconciliado con la vida, como dice el poema: "el quetuvo temor / escuchará junto a los suyos / los pasos de su madre" (Teillier, 1992: 90).

Este nivel de conciencia poética se revela claramente en el poema “ La secreta casa de la noche". En este texto la casa donde los amantes se encuentran es la casa donde el sujeto vuelve al misterioso uno primordial, como diría N ietzsche, a la unión del hombre consigo mismo y con su naturaleza. A este sujeto-personajele está dada la posibilidad del erotismo como descubrimiento dela profundidad humana. En esa casa no importa que el viento olvide el nombre del suje to, puesto que éste ya no está ahí (el nombre y la conciencia del 'yo' ya no importan), el sujeto ha dejado de ser en el nombre y en la palabra. La unión de los amantes, el espacio que los protege, "la secreta casa de la noche", constituyen la concreción de un espacio que se hace habitable en la medida que el sujeto deja de ser, en parte, ese yo que se visualiza en los anteriores textos citados. Este momento, en la poesía de Teillier, constituye una afirmación de la poesía como posibilidad de vida o como triunfo de la voluntad estética.

\footnotetext{
${ }^{10} \mathrm{Al}$ respecto, Binns lee el mismo poema en clave trágica: “El poema termina con una visión bastante ambivalente de la muerte o la no-vida (?), marcada por el aburrimiento y por una felicidad al menos cuestionada (...) La Edad de O ro vislumbrada por el poeta no corresponde a un futuro mejor, sino simplemente a una prolongación gris, trasladada al más allá, de la vida cotidiana del pueblo" (Binns, 2001: 55).
} 
Pero el lar es una construcción frágil. El lar es un espacio que nunca se termina de proyectar en el imaginario del autor. El lar como construcción humana secaracteriza según los signos de la precariedad. N ada hay de eternidad en el lar. El anhelo dela eternidad y dela permanencia es un espejismo. Por ello, el sujeto de estos poemas tiene que necesariamente devenir en lo imperceptible. Sin este devenir, el lar se convertiría en otro orden sitiado, en la ciudad triste, en la ciudad del ser deshumanizado y nihilista. Así, el sujeto que está en el lar va otra vez hacia los límites, para buscarse en el afuera de sí. Y con ello se inicia el segundo movimiento desterritorializante.

En los límites del lar se ubica la escena del sujeto del poema "Un desconocido silba en el bosque". El bosque es lo que está afuera del lar. El bosque es, a su vez, lo desconocido, la oscuridad. El desconocido que silba en el bosque es un sujeto imperceptible. Lo imperceptible no significa la nada, ni la desintegración del o los sujetos. En este caso lo imperceptible es ese sujeto desconocido, que se hace perceptible para el sujeto queasoma su oído al bosque, es decir, al afuera de todo lo poéticamente percibido. Al respecto, señalan D eleuze y Guattari: “En ese plan, no sólo se conjugan devenires-mujer, devenires-animales, deveniresmoleculares, devenires-imperceptibles, sino que lo imperceptible deviene algo necesariamente percibido" (D eleuze y Guattari, 1997: 283). En el afuera el silbido del desconocido se alcanza a oír entre la espesura del bosque, pero hay que asomar la cabeza y agudizar los sentidos: lo imperceptible es el mismo sil bido del desconocido. Ese silbido es imperceptible para el oído del forastero, por ello es necesario que el sujeto mude su propia forma de percibir el mundo que lo rodea; es necesario olvidar incluso el propio lenguaje, abandonar la "fría malla" como dice Robert Graves o la "vieja rejilla", como dice Jacques D errida. Teillier señala: "hemos cambiado de lenguaje / y nadie podrá comprender a los que oímos / a un desconocido silbar en el bosque" (Teillier, 1992: 43). Esebosquees el espacio abierto de lo imperceptible. Luego del lar ya no hay otro territorio. Afuera del lar, sólo se encuentran lugares de paso, lugares aún más precarios que la misma ciudad y que el mismo lar. U no de estos espacios es el Ilamado "H otel nube".

Sin embargo, es necesario reflexionar más sobre el lar. La crítica ha identificado la destrucción de la al dea lárica, ha señalado el devenir trágico de los lares, asociando los sujetos teillierianos con un cierto tipo de héroe del fracaso (Binns, 2001). Sin embargo, a partir de ese devenir es posible visualizar tanto un sujeto posárico en la poesía de Teillier, como otra figura del autor (también posárica) dibujada en el texto. El sujeto lárico, en este sentido, no se desintegra ni muere $y$, de esta manera, la ficción literaria traza otra figura de autor - el autor imperceptible que describe otro proceso de construcción autorial y que remite a otra manera de ser autor dentro del espacio poético. El poeta lárico -y los sujetos de 
la ficción lárica- desaparecen para dar cabida a la figura del autor imperceptible ${ }^{11}$, que implica otra conciencia y otra sensibilidad poética.

Q uizás la tragedia consista precisamente en creer en la permanencia del lar o de la palabra poética que nombra al mundo. D e modo que de ese lar el sujeto también parte, vuelve quizás a la ciudad moderna, a morir refugiado en un bar, pero también deja de ser - autor, poeta- para ser el canto mismo de las cosas, el silbido del desconocido, la voz de lo imperceptible. Así el poeta se despide de su lar, como se despide el poeta S. Esenin en su "Soy el último poeta de la aldea", que parecen ser palabras del propio Teillier: "Soy el último poeta de la aldea, I mis cantos son humildes como un puente de madera, $(\ldots)$, se extinguirá la dorada llama / de este cirio de cera humana / y el remoto reloj de la luna / gruñirá mi postrer campanada" (Teillier, 1996b: 68-69).

Afuera del lar, seha dicho, sólo hay lugares de paso. U no deestos lugares, que no son nunca de permanencia, es el "H otel nube". Al respecto, el mismo título es un indicador temático muy sugerente. El hotel es un recinto al que llegamos siempre, pero que pronto abandonamos; las nubes están siempre en movimiento como los sujetos que van de paso por el afuera. Los seres que se quedan en hoteles de uno u otro pueblo son siempresujetos que están de paso, son siempre forasteros. El sujeto transita, ihacia otra ciudad?, ihacia otra realidad?, no importa el destino, sino el tránsito mismo en el afuera, en lo desconocido. La permanencia en el hotel es precaria, de ahí que nadie pueda quedarse, de ahí que haya que inevitablemente, como única salida, como última línea de fuga, devenir en lo imperceptible.

\section{EL AUTOR IM PERCEPT IBLE}

Pero, ¿qué es finalmente este devenir imperceptible?, ¿qué pasa con el sujeto que devieneimperceptible? Respecto del concepto devenir señalan D eleuzey G uattari:

D evenir no es alcanzar una forma (identificación, imitación, mimesis), sino encontrar la zona de vecindad, de indiscernibilidad o de indiferenciación tal

\footnotetext{
${ }^{11}$ Varias son las interpretaciones que se han propuesto para este momento de la obra del poeta. La crítica ha identificado acertadamente la destrucción de la aldea lárica, lo que se traduce en una vuelta al orden dela ciudad moderna, es decir, una reterritorialización al estado sitiado. Sin embargo se visualiza otro movimiento del (o los) sujeto(s), un devenir imperceptible que proyecta y describe otra figura de autor. Ambos movimientos coexisten, forman parte del periplo que traza esta poesía Este doble movimiento se percibe en el mismo conjunto de poemas $\mathrm{H}$ otel nube. Al respecto discuto las ideas de Binns, en tanto dibujan una figura fracasada del autor, en donde los sujetos son caracterizados según su tendencia trágica y degradante o según una exagerada y continua desintegración.
} 
que ya no quepa distinguirse de una mujer, de un animal o de una molécula (D eleuze y G uattari, 1997: 11-12).

El escritor deja entonces de ser escritor para devenir otro, para devenir imperceptible. El autor, final mente, desaparece. Como lo señala M ichel Foucault (1999) en su ensayo " ¿Q ué es un autor?", el autor es quien "representa el papel del muerto en el juego de la escritura"; muertequepasa, justamente, por la "desaparición de los caracteres individuales del sujeto escritor, mediante todos los ardides que establece entreél y lo queescribe" (Foucault, 1999: 4). Así la poesía deja deser el resultado de esa concienciaindividual creativa-el autor individualizadopara ser el canto mismo de las cosas, la molécula de la poesía del afuera, su sonido imperceptible, el mismo silbido del desconocido en el bosque. Como dice el sujeto del poema "Estas palabras":

\author{
Estas palabras quieren ser \\ un puñado de cerezas, \\ un susurro - ¿para quién?- \\ entre una y otra oscuridad. \\ Sí, un puñado de cerezas, \\ un susurro - ¿ para quién ?- \\ entre una y otra oscuridad. \\ (Teillier, 1996a: 10 ).
}

El carácter metapoético del texto nos habla de una nueva forma de entender la misma poesía y condición autorial del escritor. En la expresión del deseo se encuentra el devenir impercepti ble de la misma palabra poética. El susurro, que quieren ser las palabras, es el susurro de lo imperceptible, el puñado de cerezas sobre la mano de un sujeto a la vez imperceptible; ese sujeto en el que deviene el autor para, final y felizmente, desaparecer en su poesía.

El autor juega a desaparecer en la obra, y de este modo la propia obra - en la ficción poética- deviene espacio simulado de muerte, espacio donde tiene que desaparecer el autor para afirmar su apuesta dejuego: su obra que lo hace ser tal. Como dice Foucault:

La escritura se despliega como un juego que infatigablemente va siempre más allá de sus reglas, y de este modo pasa al exterior. En la escritura, no se trata de la manifestación o exaltación del gesto de escribir; no se trata de la sujeción de un sujeto (el autor) a un lenguaje: se trata de la apertura de un espacio en donde el sujeto que escribe no deja de desaparecer (Foucault, 1999: 4).

D eesta forma, el proyecto poético ya no se percibecomo la construcción de 
un espacio, sino como el devenir mismo de la poesía en lo imperceptible, porque como dicen $D$ el euze y $G$ uattari "escribir también es devenir otra cosa que escritor" (D eleuze y Guattari, 1997: 17). La fuga se constituye en el momento en queel autor deviene imperceptible, a través de la anulación de todo yo discursivo. Esta línea de fuga alcanza su máxima expresión en el poema "Bienes".

En "Bienes", mediante el devenir imperceptible del sujeto dela enunciación, se devi ene finalmentemolécula. $\mathrm{N}$ ada hay de un yo o de un tú en el poema, tampoco se trata de una simple enumeración de objetos, al parecer, de poca importancia. Los objetos que nombran han perdido su calidad material, vienen a repre sentar el espíritu ahora (im)perceptible del poeta a través de las cosas que valora y canta, queadquieren un valor de talismanes: losúnicos "Bienes" queverdaderamente se pueden desear en el afuera de lo imperceptible.

D e estos objetos cabe mencionar algunos, quizás los más sugerentes: "Un libro de Edgar Poe", el libro, único compañero del viaje; "un pasaje de tren", es decir, la posibilidad del viaje abierto hacia lo distinto, la línea de fuga que traza el recorrido del tren siempre hacia otro lugar, hacia otra estación; "un Ilavero sin llaves", puesto que en el viaje por el afuera nunca habrá una puerta de una casa para abrir; por último, "un poema inconcluso", ya queel verdadero poema nunca se ha escrito, siempre se está escribiendo, ya que el poema nunca es algo que es ni que será - como dice H uidobro- sino que está en perpetua formación, en continuo cambio, en devenir constante, tal como la propia figura del autor.

D evenir imperceptible no significa entonces "el fin de la poesía" o "la muerte del autor", al contrario, se presenta como una dester ritorialización total que permite al sujeto autor la reconciliación con la vida entendida como existencia poética a través del canto mismo de las cosas. El ser desaparece así en sustancia poética y en ella se oculta, sin dejar de ser. Este devenir imperceptible constituye, a mi parecer, la concreción del proyecto poético deTeillier. El "real ismo secreto" como expresión poética, perseguido en la proyección del lar, se explicita aquí, a partir de $\mathrm{H}$ otel nube, mediante el devenir imperceptible del sujeto de esta poesía; devenir que traza una figura imperceptible de autor, a la cual le está dado ser acogida secretamente en la naturaleza de las cosas, en la medida en que de sí mismo se ha abandonado y en la medida en que todo espacio ha dejado de proyectarse en tanto orden perdurable.

La escritura de Teillier traza, entonces, un periplo hacia el afuera del yo. EI sujeto abandona la ciudad donde se ha abandonado, se refugia en las estaciones a esperar un tren hacia otra estación sin nombre, acaso intuye que pertenece a su verdadero espacio: el afuera, donde sólo se es un forastero. Cerca de la frontera el lar lo ha estado esperando; es necesario construir la casa, buscar al cuerpo amado del otro, volver a la vida, reconciliarse con la naturaleza. Sin embargo, no existe afuera la permanencia, el lar también es el pueblo del olvido, la aldea 
lárica se destruye, la ilusión de la eternidad de la "Edad de oro" se desvanece y sólo quedan sombras. Pero el afuera, el bosque próximo a la casa triste, el afuera de lo impercepti ble es un espacio para abandonarse felizmente. El sujeto imperceptible devi ene canto, susurro de la naturaleza, sonido de agua en las acequias, silbido de un desconocido en el bosque, frutos empuñados en la mano de alguien. El sujeto sin nombre es ahora la voz de lo imperceptible, la voz que, a su vez, es el canto de las cosas.

Ese sujeto describe otra figura y otra conciencia de autor. Un autor imperceptible, pero percibido en la marca de la desaparición del autor, en la huella de la ausencia. Ese autor dibuja un complejo proceso de construcción autorial que se revela como una unidad en constante creación asociada al cuerpo-autor que identificamos con el nombre de JorgeTeillier. Así, la poesía deTeillier expresa la necesidad de una conciencia poética del mundo, del propio autor en el mundo y de un orden más armónico, donde los poetas imperceptibles - pero perceptibles aun en su devenir- son, finalmente, los "simples hermanos de los seres y las cosas" (Teillier, 1999: 24).

\section{REFERENCIAS BIBLIOGRAFICAS}

Aldunate, Pedro. 2004. "La construcción autorial de Armando U ribe Arce. El autor, la obra y la muerte". Tesis deM agíster en Literatura $\mathrm{H}$ ispanoamericana, U niversidad de Concepción. Concepción, Chile.

Barthes, Roland. 1981. Crítica y verdad. Buenos Aires: Siglo XXI Argentina Editores S.A.

Binns, N iall. 2001. La poeśa de Jorge Teillier: La tragedia de los lares. Concepción, Chile: Ediciones Lar.

Blanchot, M aurice. 1993. D eKafka a Kafka. Buenos Aires: Fondo de Cultura Económica de Argentina, S.A.

Contreras, M arta. 1994. G riselda Gambaro. Teatro dela descomposición. Concepción, Chile: Ediciones U niversidad de Concepción.

D eleuze, Gilles. 1994. La literatura y la vida. Córdoba: Alción Editora.

D eleuze, Gilles y G uattari, Félix. 1990. Kafka: por una literatura menor. M éxico, D .F.: Ediciones Era, S.A.

. 1997. M il mesetas. Valencia: Editorial Pretextos.

D errida, Jacques. 1971. D ela gramatología. Buenos Aires: Siglo XXI Argentina Editores S.A.

Espinoza, Christian. 2001. La mirada escénica (Glosa ). C oncepción, Chile: Universidad de Concepción.

Fou cault, M ichel. 1999. "Q ué es un autor", en Literatura y conocimiento. En: http:// www.saber.ula.ve/db/ssaber/Edocs/centros investigacion/csi/publicaciones/papers/ davila-autor.pdf 
García C anclini, N éstor. 1989. Culturas híbridas, etrategias para entrar y salir de la modernidad. M éxico, D.F.: Editorial Grijalbo.

Gomes, M iguel. 2003. Seminario de Escuela de Verano "Los mitos del autor en la literatura latinoamericana", M agíster en Literatura H ispanoamericana, U niversidad de Concepción.

H ozven, Roberto. 1979. El estructuralismo literario. Santiago, C hile: D epartamento de Estudios H umanísticos.

Kristeva, Julia. 1981. Semiótica I y II. Barcelona: Editorial Fundamentos.

M uñoz, Luis y 0 elker, Dieter. 1995. D iccionario de movimientos y grupos literarios. Concepción, Chile: Ediciones U niversidad de Concepción.

N ietzsche, Friedrich. 1951. El origen de la tragedia. Buenos Aires: Aguilar Editores.

Pavis, Patrice. 1998. D iccionario del teatro: D ramaturgia, estética, semiología. Barcelona: Ediciones Paidós I bérica, S.A.

Santibáñez, Cristián. 2004. "N otas sobre el autor y su función”, en Acta Literaria N o 29, pp. 135-147.

Teillier, Jorge. 1992. Los dominios perdidos. M éxico, D .F.: Fondo de Cultura Económica, S.A.

- - - 1996a. H otel nube Concepción, C hile: Ediciones Lar.

- - - 1996b. Poeśa U niversal traducida por poetas chilenos. Santiago, Chile: Editorial Universitaria.

- - - 1999. Prosas Santiago, Chile: Editorial Sudamericana C hilena.

Zapata, Juan. 2005. Seminario "Lírica chilena contemporánea”, D octorado en Literatura Latinoamericana, Universidad de Concepción, Chile. 